\title{
CFD Simulation for Separation of Carbon Dioxide-Methane Mixture by Pressure Swing Adsorption
}

\author{
K. Rambabu, L. Muruganandam, and S. Velu \\ School of Mechanical and Building Sciences, VIT University, Vellore, Tamil Nadu 632014, India \\ Correspondence should be addressed to K. Rambabu; rambabu.k@vit.ac.in
}

Received 13 January 2014; Revised 28 May 2014; Accepted 29 May 2014; Published 19 June 2014

Academic Editor: Donald L. Feke

Copyright (C) 2014 K. Rambabu et al. This is an open access article distributed under the Creative Commons Attribution License, which permits unrestricted use, distribution, and reproduction in any medium, provided the original work is properly cited.

\begin{abstract}
A developing technology for gas separations is pressure swing adsorption, which has been proven to be more economical and energy efficient compared to other separation methods like cryogenic distillation and membrane separation. A pressure swing adsorption (PSA) column, with carbon dioxide-methane as feed mixture and 6-FDA based polyimides as the adsorbent, was modeled and simulated in this work. Ansys Fluent 12.1, along with supplementary user defined functions, was used to develop a 2D transient Eulerian laminar viscous flow model for the PSA column. The model was validated by comparing the simulated results with established analytical models for PSA. The developed numerical model was used to determine the carbon dioxide concentration in the column as a function of time based on different operating conditions. Effect of various operating parameters like pressure, temperature, and flow rate on the separation efficiency has been studied and reported. Optimization studies were carried out to obtain suitable operating conditions for the feed gases separation. Simulation studies were carried out to determine the separation length required for complete separation of the feed mixture corresponding to different inlet feed concentrations which were entering the column at a given flow rate.
\end{abstract}

\section{Introduction}

Traditionally, industrial gas separation is done by chemical and physical absorption, membrane separation, and low temperature distillation [1]. Owing to high energy consumption and associated problems like cost and process degradation, newer avenues are being explored. With different kinds of adsorbents available, each offering a wide range of equilibrium properties, adsorption is being seen as a viable option for gas separation, especially for binary gas mixtures [2]. Separation processes utilizing adsorption are low cost and energy efficient alternatives in comparison with other separation processes particularly at low to moderate throughput [3].

Adsorption is mainly performed by periodic variations of pressure and temperature of the system. Temperature swing adsorption (TSA) is widely used for removal of trace amounts of pollutants such as volatile organic compounds from gaseous streams [4]. However, TSA needs precise process controls, huge columns, and longer operating time compared to pressure swing adsorption (PSA) and hence, TSA is preferred for systems having strong adsorbing nature with respect to the adsorbent [5].

Pressure swing adsorption is based on Skarstrom cycle [6] in which feed mixture is sent in at high pressures. Separation takes place due to selective adsorption of one or more feed components by the adsorbent and the rate of adsorption is regulated by the operating pressure of the process. The adsorbed component (adsorbate) is recovered later by reducing the pressure of the column. Adsorption involves contacting a free fluid phase (gas or liquid) with a rigid particulate phase which has the property of selectively taking up and storing one or more solute species originally contained in the fluid [7]. The strength of the surface interaction between the adsorbent and adsorbate depends on the nature of the solid adsorbent, the fluid adsorbate, and the system's thermodynamic properties, especially density and pressure.

Consequently, different adsorbents adsorb feed components with different affinities which are regulated by 
the thermodynamics of the system. This gives raise to the wide range of processes in PSA processes with respect to the adsorbent used.

Two separations of particular commercial interest that have been mostly reported in the literature are the carbon dioxide-methane $\left(\mathrm{CO}_{2}-\mathrm{CH}_{4}\right)$ separation and oxygenair separation. Separation of oxygen from air by PSA using zeolite $5 \mathrm{~A}$ as adsorbent has been studied experimentally $[8,9]$. Modelling of the oxygen separation from air by PSA using $13 \mathrm{X}$ zeolite as adsorbent has been carried out by equilibrium model with a linear isotherm for oxygen and a polynomial isotherm for nitrogen [10]. Experimental studies on the separation of the carbon dioxide-methane system using membrane technology have been reported in scientific literature [11]. Simulation study on the effect of gas solubility in the adsorbent with respect to feed mixture separation is also made for $\mathrm{CO}_{2}-\mathrm{CH}_{4}$ system using PSA [12]. However, the effect of operating parameters on the separation efficiency of the system has not been explored so far.

Removal of carbon dioxide from natural gas is extremely important in order to meet the transportation pipeline specifications as well as produce natural gas from associated gas dug out from oil wells [11, 13]. The most common adsorbents used in PSA separation processes for carbon dioxide-methane system are inorganic adsorbents like zeolite, activated charcoal, and polymer adsorbents like polysulfone and polyacetate. The 6-FDA based polyimides have been reported to be a promising adsorbent material for gas separation [14]. The hexafluoroisopropylidene linkage in the polyimides enhances the permselectivity of the 6-FDA based polyimides for the binary systems [15]. Polyimides also show excellent mechanical strength as well as temperature and chemical resistance [16].

In this study, the separation process of $\mathrm{CO}_{2}-\mathrm{CH}_{4}$ system was modeled based on dynamic pressure-swing adsorption process with FDA based polyimides particles as adsorbent. CFD package, Ansys Fluent 12.1, along with supplementary user defined functions was used to develop a $2 \mathrm{D}$ transient Eulerian laminar viscous flow model for the adsorption column. The objective of the investigation was to simulate the developed numerical model to study the individual feed component's concentration distribution and separation efficiency with respect to time and column operating parameters.

\section{Process Modelling}

2.1. Model Setup and Analysis. A typical model of the PSA adsorption column is shown in Figure 1. The model was developed and simulated using Ansys Fluent 12.1. The details of the adsorption column model were taken as used in earlier related work [12] and are shown in Table 1. The fluid was taken to be incompressible, Newtonian, and in a laminar flow regime. Carbon dioxide-methane system at standard conditions was chosen as the feed mixture in a 50\%-50\% volume concentration. The porous medium approach was used in the simulation of the fluid flow in the packed bed since the adsorbent inside the packed bed can be treated as porous medium. The porous medium is assumed to

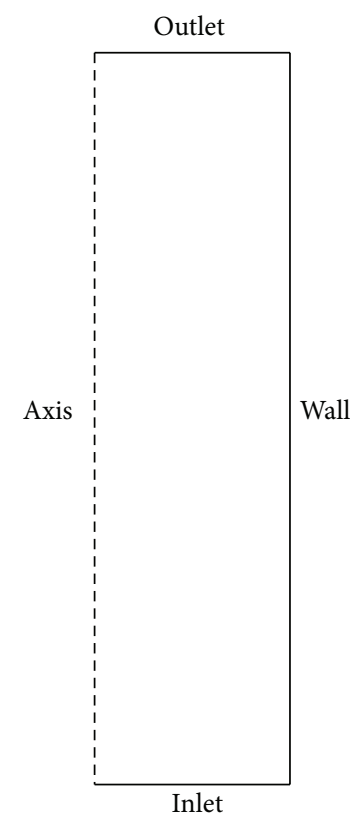

FIGURE 1: Schematic of the PSA column model with feed inlet and outlet.

TABLE 1: Parameters used in separation of binary gas mixtures.

\begin{tabular}{lc}
\hline Molar feed composition & $\mathrm{CO}_{2}-\mathrm{CH}_{4}(50 \%-50 \%$ by volume) \\
Adsorbent & Polyimide (6FDA-ODA) \\
Particle radius, $\mu \mathrm{m}$ & 100 \\
Bed length, $\mathrm{m}$ & 0.35 \\
Bed diameter, $\mathrm{m}$ & 0.06 \\
Bed voidage & 0.5 \\
Operating temperature, $\mathrm{K}$ & 300 \\
Operating pressure, atm & 20 \\
Glass transition temperature & $\sim 450 \mathrm{~K}$ \\
\hline
\end{tabular}

be isotropic. The approach uses an additional mass source term to the standard fluid flow equations to account for the flow resistance due to the porous medium. The mixing and transport of binary species are modeled by solving conservation equations describing convection and diffusion for each component species. The following assumptions have been made in the modelling approach.

(i) The process is assumed to be isothermal.

(ii) Ideal gas law is applicable.

(iii) Plug flow is assumed; that is, there is no axial or radial dispersion.

(iv) The mass transfer rate is given by linear driving force (LDF) model.

User defined functions were introduced to incorporate source terms in order to include the effects of solubility and diffusivity. The effect of temperature on permeability was incorporated in the source term by (1) using the data given in Table 2 [17]. PISO scheme was used as the pressure velocity coupling algorithm. The governing equation for fluid flow 
TABLE 2: Activation energies for permeation for 6-FDA based membranes at $35^{\circ} \mathrm{C}$ and $20 \mathrm{~atm}$.

\begin{tabular}{lcc}
\hline & $\mathrm{CO}_{2}$ & $\mathrm{CH}_{4}$ \\
\hline Kinetic diameter $(\AA)$ & 3.30 & 3.80 \\
$\lambda_{0}($ Barrer $)$ & 17.25 & 0.20 \\
$E_{p}(\mathrm{kcal} / \mathrm{mol})$ & 4.74 & 0.92 \\
\hline
\end{tabular}

in isotropic porous media is given by (2). Incorporating the source term, $S_{\emptyset}$, in (2) would enable to define concentration points of adsorbed gas. Thus, the mass and momentum conservation equations for the discussed model are given by (3) and (4), respectively. Inertial and viscous resistance encountered by the fluid are incorporated in the model by the last two terms. Permeability $(\alpha)$ is calculated using Darcy's law (see equation (5)) due to the presence of porous media. The linear driving force model describing the mass transfer phenomena for PSA is given by (6) and (7). The governing equations were discretized by second-order upwind scheme:

$$
\begin{gathered}
P=\lambda_{o} \exp \left(\frac{E_{P}}{R T}\right), \\
\frac{\partial(\gamma \rho \Phi)}{\partial t}+\nabla \cdot(\gamma \rho \vec{V} \Phi)=\nabla \cdot(\gamma \Gamma \nabla \Phi)+\gamma S_{\Phi}, \\
\frac{\partial(\gamma \rho)}{\partial t}+\nabla \cdot(\gamma \rho \vec{V}) \pm \nabla \cdot\left(\varepsilon D_{p, i} \nabla \cdot(\gamma \rho)\right)=0, \\
\frac{\partial(\gamma \rho \vec{V})}{\partial t}+\nabla \cdot(\gamma \rho \vec{V} \vec{V}) \\
=-\gamma \nabla P+\nabla \cdot(\gamma \vec{\tau}) \\
+\gamma \overrightarrow{B_{f}}-\left(\frac{\mu}{\alpha}+\frac{C_{2} \rho}{2}|\vec{V}|\right) \vec{V}, \\
\alpha=\frac{d_{p}^{2} \varepsilon^{3}}{150(1-\varepsilon)^{2}}, \\
\frac{\partial q_{i}}{\partial t}=k_{i}\left(q_{i}^{*}-q_{i}\right), \\
k_{i}=\frac{60 D_{p, i}}{d_{p}^{2}} .
\end{gathered}
$$

2.2. Model Validation. The adsorption of small molecules in glassy polymers is described best by the dual mode (DM) sorption model $[18,19]$. The dual sorption mechanism is given as a combination of Henry's law of solubility and Langmuir adsorption [18]. Henry's law accounts for the dissolution of the penetrant molecule in the continuous polymer chain matrix and its sorption in microvoids is described by Langmuir model. According to DM sorption model, the gas concentration in the polymer is given by the following equation as a function of operating pressure:

$$
C=C_{D}+C_{H}=k_{D} p+\frac{C_{H}^{\prime} b p}{(1+b p)}
$$

TABLE 3: DM sorption model parameters for carbon dioxide sorbed in polyimide.

\begin{tabular}{lc}
\hline$k_{D}\left(\mathrm{~cm}^{3}\right.$ of $\mathrm{CO}_{2} / \mathrm{cm}^{3}$ of adsorbent bar $)$ & 1.56 \\
$C_{H}^{\prime}\left(\mathrm{cm}^{3}\right.$ of $\mathrm{CO}_{2} / \mathrm{cm}^{3}$ of adsorbent $)$ & 54.6 \\
$b\left(\mathrm{bar}^{-1}\right)$ & 0.544 \\
\hline
\end{tabular}

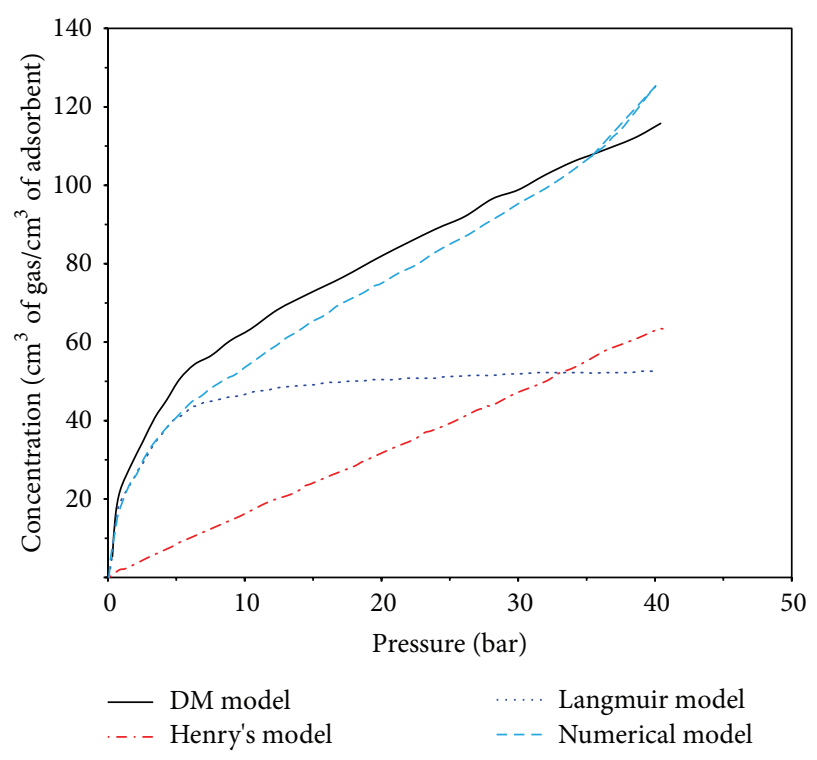

FIGURE 2: Comparison of DM model and numerical model.

where $C$ is the concentration of the gas adsorbed in unit volume of the polymer, $C_{D}$ is the gas concentration by dissolution, $C_{H}$ is the gas concentration by hole filling, $p$ is the applied pressure, $k_{D}$ is dissolution constant, $C_{H}^{\prime}$ is the hole saturation constant, and $b$ is the affinity constant.

DM model assumes that both modes occur simultaneously and are always in equilibrium $[18,20]$. The diffusion coefficient is independent of concentration. The values of the parameters for the DM sorption model for carbon dioxide sorbed in polyimide are specified in Table 3 [21].

Validation of the numerical model was conducted by studying the steady state adsorbed $\mathrm{CO}_{2}$ concentration as a function of operating pressure and comparing with the $\mathrm{CO}_{2}$ concentration obtained using analytical DM model. As shown in Figure 2, the behaviour of the numerical model is in accordance with the analytical DM sorption model. The variation in the amount of $\mathrm{CO}_{2}$ adsorbed per unit volume of adsorbent between the DM model and numerical model reported an average error of $8.7 \%$ (in magnitude), calculated over the operating pressure for the adsorption column. The error between the DM model and the proposed numerical model could be due to the truncation, discretization, and round-off phenomena in the simulation. The deviation between numerical model and the DM model was very much less in comparison to the deviation between Langmuir model and the DM model (average error of $42.5 \%$ ) and the deviation between Henry model and the DM model (average error of $34.9 \%)$. 


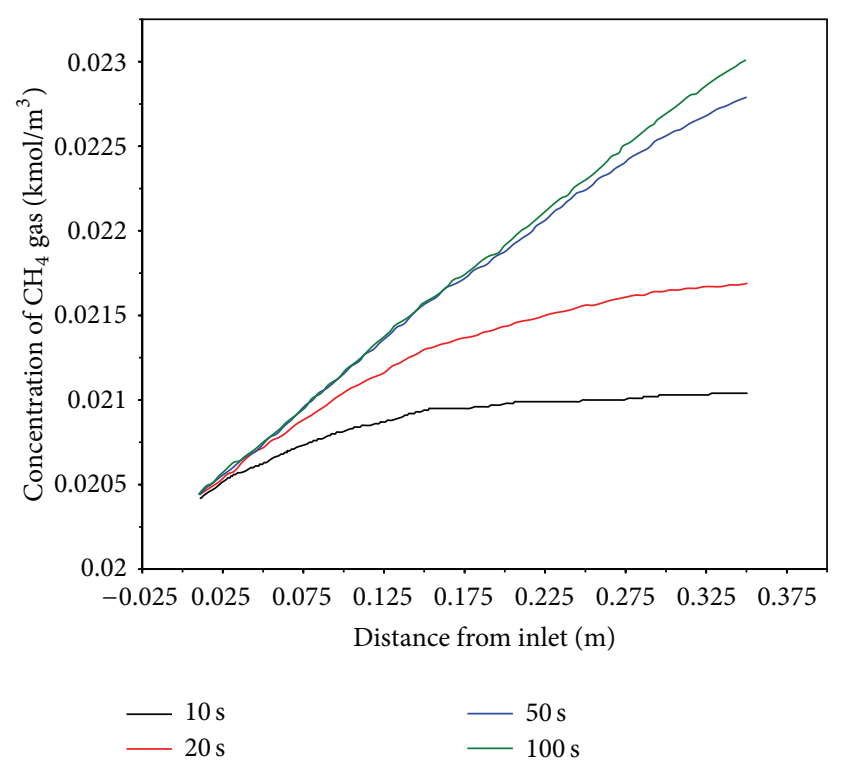

Figure 3: Concentration of methane as a function of time.

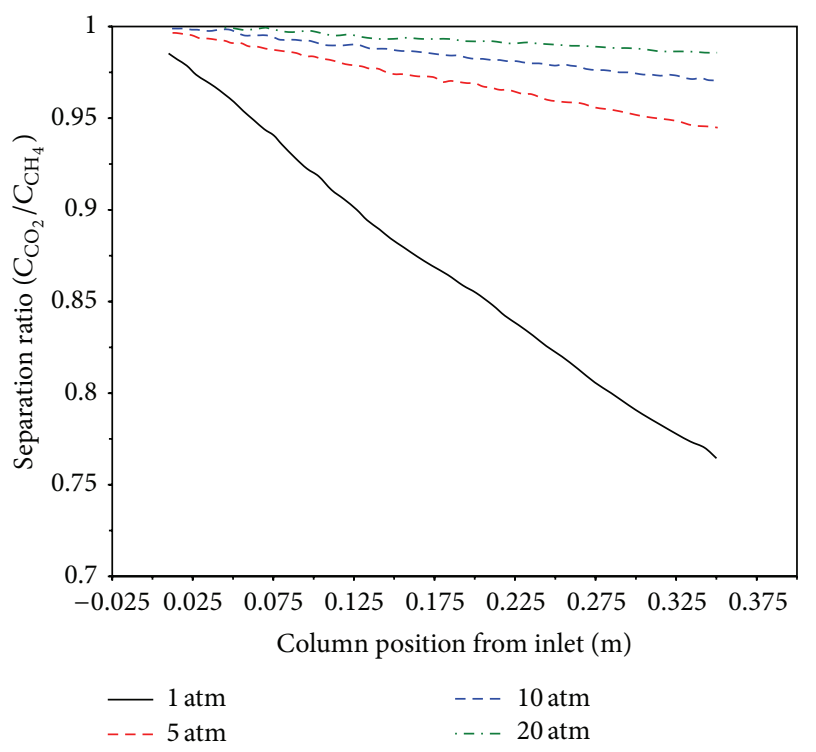

Figure 4: Pressure effects on separation ratio (flow velocity = $0.15 \mathrm{~m} / \mathrm{sec}$ and temperature $=200 \mathrm{~K})$.

2.3. Simulation. Carbon dioxide-methane mixture at a volume composition of $50 \%-50 \%$ was given as the inlet feed stream. At the end of the cycle the mole fraction of carbon dioxide was reduced to 0.05 from 0.5 due to the adsorption of carbon dioxide in the column. The result of the unsteady state simulation is presented in Figure 3. It is evident from Figure 3 that the concentration of methane at a given spatial position increased with time of adsorption. It was also evident that the rate of adsorption at a given point in the column decreased with the time of adsorption. This was due to the decreasing concentration gradient between the feed stream and the adsorbent material.

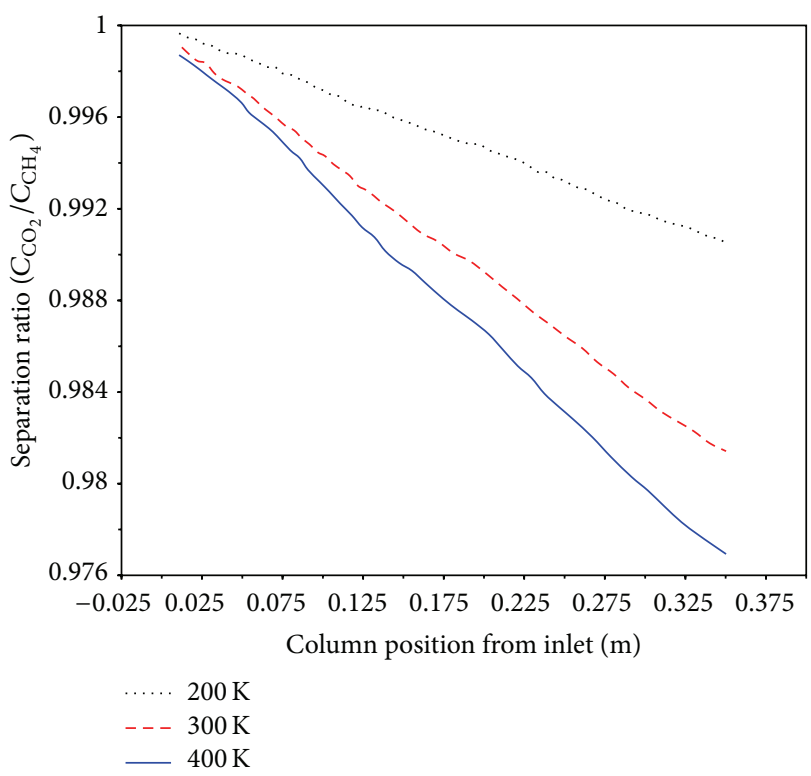

FIGURE 5: Temperature effects on separation ratio (flow velocity $=$ $0.15 \mathrm{~m} / \mathrm{sec}$ and pressure $=15 \mathrm{~atm})$.

2.3.1. Effect of Pressure. Simulation results showed that decreasing the operating pressure leads to better separation. As shown in Figure 4, the separation ratio (ratio of concentration of $\mathrm{CO}_{2}$ to concentration of $\mathrm{CH}_{4}$ ) decreased along the length of the column as pressure was reduced from 15 atm to $1 \mathrm{~atm}$.

2.3.2. Effect of Temperature. For most gases, solubility increases with increase in temperature till the glass transition temperature is reached. Simulations were carried out at $200 \mathrm{~K}, 300 \mathrm{~K}$, and $400 \mathrm{~K}$ keeping the pressure constant. It was observed that separation increases with increase in temperature. The temperature effect on the adsorption is shown in Figure 5.

2.3.3. Effect of Flowrate. Flowrate of the feed stream is an important factor affecting the separation process. The model was simulated for three different flow rates- $0.1 \mathrm{~m} / \mathrm{s}, 0.01 \mathrm{~m} / \mathrm{s}$, and $0.001 \mathrm{~m} / \mathrm{s}$. The highest degree of separation was obtained at $0.001 \mathrm{~m} / \mathrm{s}$, as evident from Figure 6 .

2.4. Optimization. The optimum operating conditions for carrying out the pressure swing adsorption for the separation of carbon dioxide-methane mixture at a volume composition of $50 \%-50 \%$ entering the column at a velocity of $0.001 \mathrm{~m} / \mathrm{s}$ was obtained by simulating the model at different operating pressures and temperatures. Pressure was reduced from $20 \mathrm{~atm}$ to $1 \mathrm{~atm}$ and the temperature dependence study was performed for $200 \mathrm{~K}, 300 \mathrm{~K}$, and $400 \mathrm{~K}$. For every run, the separation ratio was calculated and plotted to find the optimum conditions for operating the PSA column. The results are presented in Figure 7 . The optimization study revealed that a separation length of about $0.125 \mathrm{~m}$ guaranteed $100 \%$ 


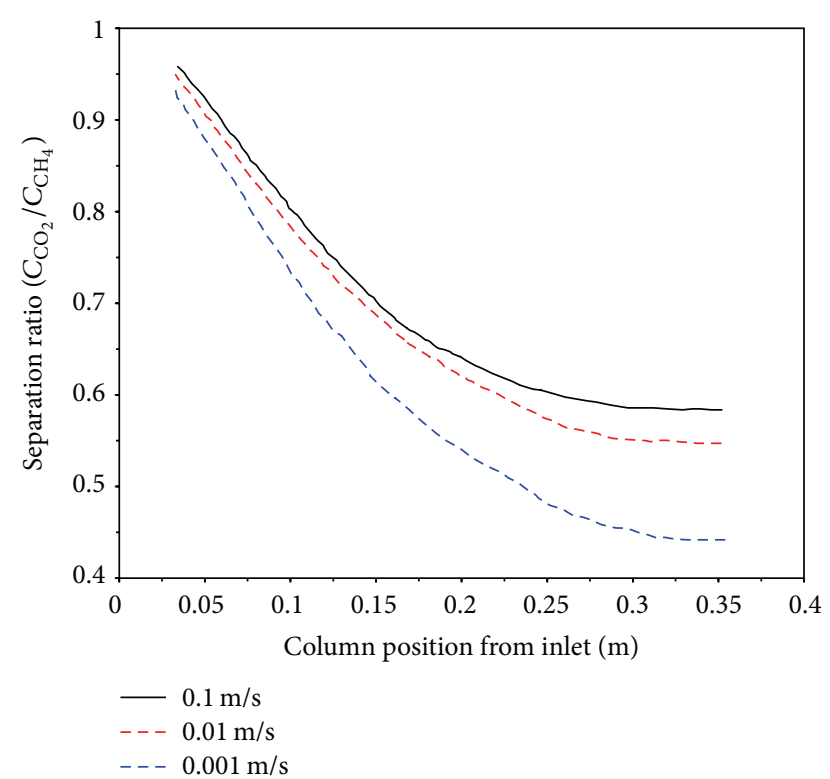

FIGURE 6: Effect of feed velocity on the separation ratio (pressure = $5 \mathrm{~atm}$ and temperature $=200 \mathrm{~K}$ ).

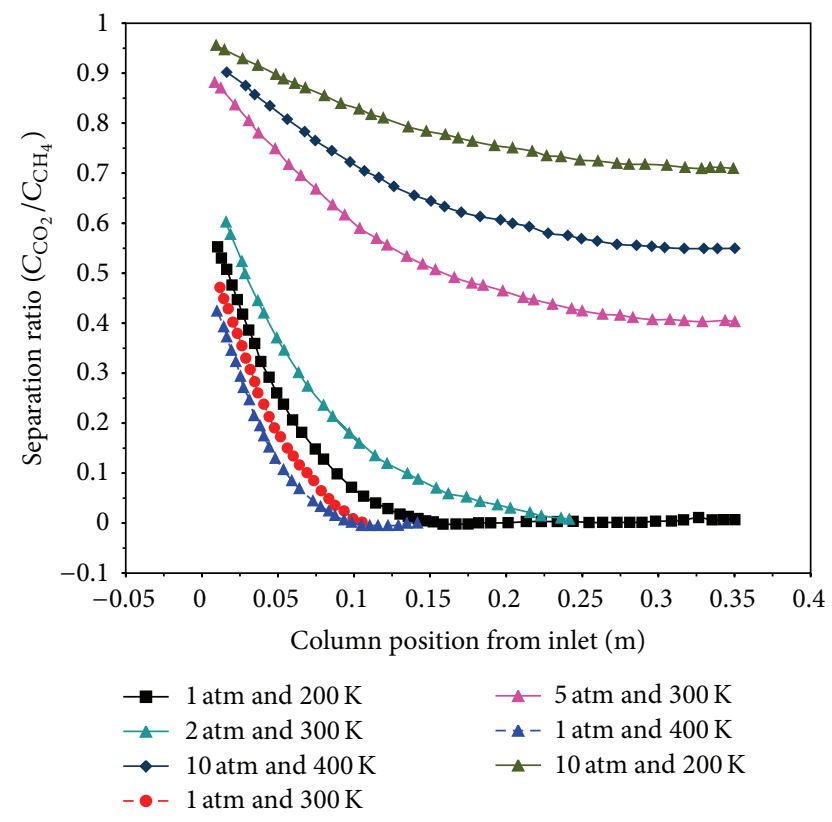

FIGURE 7: Optimization studies on separation ratio on operation conditions (feed velocity $=0.001 \mathrm{~m} / \mathrm{s}$ ).

separation for the specified feed inlet conditions. Optimum operating conditions were found to be $1 \mathrm{~atm}$ and $400 \mathrm{~K}$.

Further, as a comparative study, simulations were carried out to determine the separation length required for complete separation of the feed corresponding to different initial concentrations of the feed mixture. The results obtained are presented in Table 4.

As a future scope of study for the pressure swing adsorption using 6-FDA-ODA particles bed, a more improved

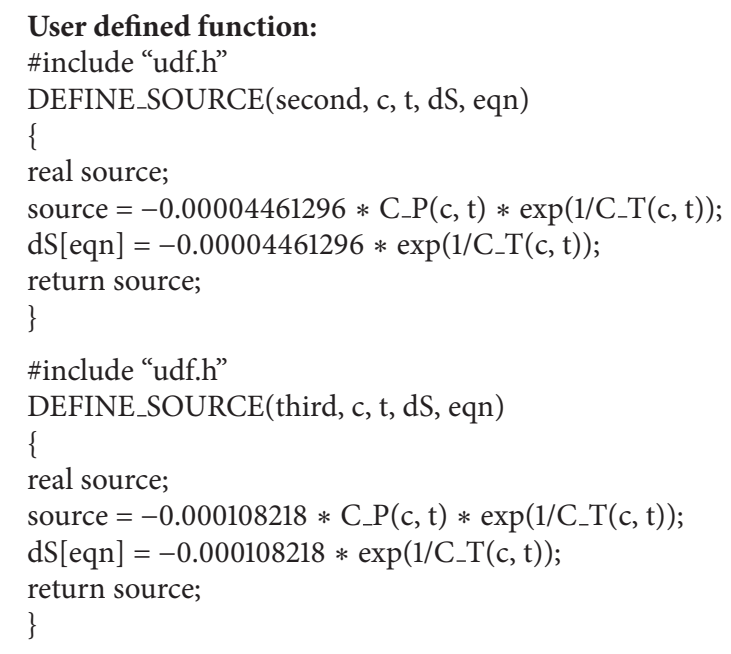

Algorithm 1: User defined function.

model (3-dimensional) incorporating most of the real time correlations, radial variation studies and temperature dependence studies for the adsorption process are to be carried out.

\section{Conclusions}

A CFD model based on pressure swing adsorption was developed for binary gas mixture separation, namely, $\mathrm{CO}_{2}$ $\mathrm{CH}_{4}$, using 6-FDA based polyimides as adsorbent. The model was validated against the analytical dual mode sorption model for adsorption of gases $\left(\mathrm{CO}_{2}\right)$ on glassy polymers. The model was used to determine the carbon dioxide concentration in the column as a function of time based on different operating conditions. Analysis clearly indicated that providing an optimal residence time by lowering the velocity of the flow produces the maximum separation efficiency. For a fixed column length of $0.035 \mathrm{~m}$, an optimal residence time of 50 seconds corresponding to a flow velocity of $0.001 \mathrm{~m} / \mathrm{s}$ yielded a separation efficiency of around $97 \%$. Lowering the operating pressure leads to better separation as permeability of gases is inversely proportional to the operating pressure. Increasing the temperature of the adsorption bed produced better separation of the gases. As an outcome of optimization studies, a reference table specifying the separation length required for complete separation of the feed mixture entering the adsorption column at different initial concentrations (with a flow velocity of $0.001 \mathrm{~m} / \mathrm{s}$ ) was also presented.

\section{Appendix}

Algorithm 1: User defined function.

\section{Nomenclature}

$P: \quad$ Total bed pressure $(\mathrm{kPa})$

$\lambda_{o}$ : Permeability (Barrer)

$E_{p}$ : Activation energy $(\mathrm{kJ} / \mathrm{mol})$ 


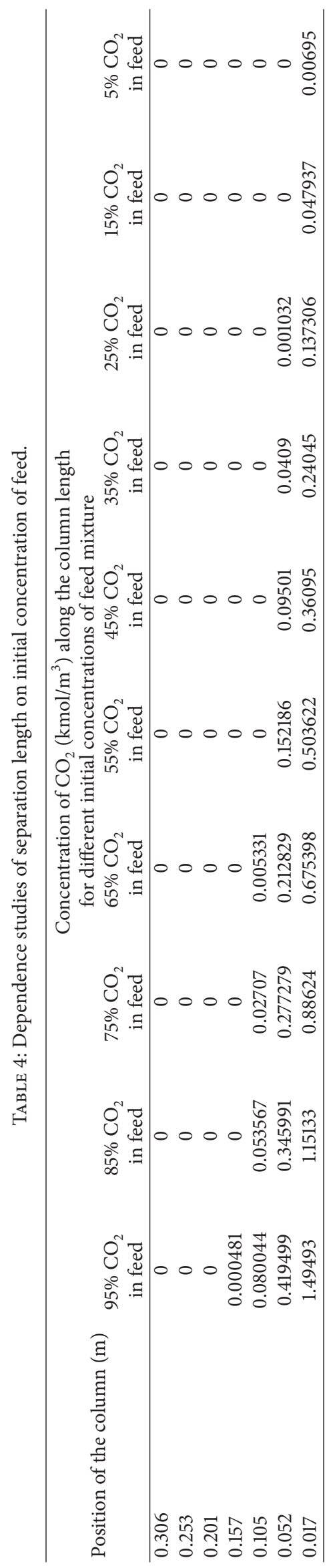


$R: \quad$ Universal gas constant $(\mathrm{J} / \mathrm{gmol} \mathrm{K})$

$\rho: \quad$ Density $\left(\mathrm{kg} / \mathrm{m}^{3}\right)$

$\gamma$ : Volume $\left(\mathrm{m}^{3}\right)$

$\vec{V}: \quad$ Velocity $(\mathrm{m} / \mathrm{sec})$

$\Phi: \quad$ Any given property

$\overrightarrow{B_{f}}:$ Body forces per unit volume $\left(\mathrm{N} / \mathrm{m}^{3}\right)$

$T: \quad$ Gas-phase temperature in the bed $(\mathrm{K})$

$\vec{\tau}: \quad$ Fluid stress $\left(\mathrm{N} / \mathrm{m}^{2}\right)$

$\mu$ : $\quad$ Absolute viscosity $(\mathrm{kg} / \mathrm{m} \mathrm{sec})$

$\alpha$ : Gas permeability (Barrer)

$d_{p}:$ Particle diameter $(\mathrm{m})$

$\varepsilon: \quad$ Bulk void fraction

$q_{i}: \quad$ Solid-phase concentration of $i$ th component $(\mathrm{gmol} / \mathrm{kg})$

$q_{i}^{*}:$ Equilibrium solid-phase concentration of $i$ th component $(\mathrm{gmol} / \mathrm{kg}$ )

$k_{i}$ : LDF model mass transfer coefficient of $i$ th component $(1 / \mathrm{sec})$

$D_{p, i}:$ Diffusivity of $i$ th component $\left(\mathrm{m}^{2} / \mathrm{sec}\right)$

$t: \quad$ Time (sec)

$b$ : Affinity constant $\left(\mathrm{bar}^{-1}\right)$

C: Gas-phase concentration of a given component $\left(\mathrm{cm}^{3}\right.$ of adsorbate $/ \mathrm{cm}^{3}$ of adsorbent)

$C_{D}$ : Gas concentration by dissolution $\left(\mathrm{cm}^{3}\right.$ of adsorbate $/ \mathrm{cm}^{3}$ of adsorbent)

$C_{H}$ : Gas concentration by hole filling $\left(\mathrm{cm}^{3}\right.$ of adsorbate $/ \mathrm{cm}^{3}$ of adsorbent).

\section{Conflict of Interests}

The authors declare that there is no conflict of interests regarding the publication of this paper.

\section{References}

[1] R. E. Treybal, Mass-Transfer Operations, vol. 3, McGraw-Hill, New York, NY, USA, 1980.

[2] C. M. Yon and J. D. Sherman, Adsorption, Gas Separation, KirkOthmer Encyclopedia of Chemical Technology, 2004.

[3] D. Aaron and C. Tsouris, "Separation of $\mathrm{CO}_{2}$ from flue gas: a review," Separation Science and Technology, vol. 40, no. 1-3, pp. 321-348, 2005.

[4] F. I. Khan and A. K. Ghoshal, "Removal of Volatile Organic Compounds from polluted air," Journal of Loss Prevention in the Process Industries, vol. 13, no. 6, pp. 527-545, 2000.

[5] M. A. Kalbassi, R. J. Allam, and T. C. Golden, "Temperature swing adsorption," U.S. Patent no. 5,846,295, U.S. Patent and Trademark Office, Washington, DC, USA, 1998.

[6] C. W. Skarstrom, "Method and apparatus for fractionating," U.S. Patent no. 2,944,627, U.S. Patent and Trademark Office, Washington, DC, USA, 1970.

[7] A. Kapoor and R. T. Yang, "Kinetic separation of methanecarbon dioxide mixture by adsorption on molecular sieve carbon," Chemical Engineering Science, vol. 44, no. 8, pp. 1723$1733,1989$.

[8] A. M. Mendes, C. A. Costa, and A. E. Rodrigues, "Oxygen separation from air by PSA: modelling and experimental results: part I," Separation and Purification Technology, vol. 24, no. 1-2, pp. 173-188, 2001.

[9] S. Farooq, D. M. Ruthven, and H. A. Boniface, "Numerical simulation of a pressure swing adsorption oxygen unit," Chemical Engineering Science, vol. 44, no. 12, pp. 2809-2816, 1989.

[10] J. C. Kayser and K. S. Knaebel, "Pressure swing adsorption: development of an equilibrium theory for binary gas mixtures with nonlinear isotherms," Chemical Engineering Science, vol. 44, no. 1, pp. 1-8, 1989.

[11] S. Sridhar, B. Smitha, and T. M. Aminabhavi, "Separation of carbon dioxide from natural gas mixtures through polymeric membranes-a review," Separation \& Purification Reviews, vol. 36, no. 2, pp. 113-174, 2007.

[12] H. S. Pierre, Simulation of dynamic pressure-swing gas sorption in polymers [Ph.D Dissertation], 2005.

[13] S. Li, J. G. Martinek, J. L. Falconer, R. D. Noble, and T. Q. Gardner, "High-pressure $\mathrm{CO}_{2} / \mathrm{CH}_{4}$ separation using SAPO-34 membranes," Industrial \& Engineering Chemistry Research, vol. 44, no. 9, pp. 3220-3228, 2005.

[14] H. Hachisuka, T. Ohara, and K. Ikeda, "New type asymmetric membranes having almost defect free hyper-thin skin layer and sponge-like porous matrix," Journal of Membrane Science, vol. 116, no. 2, pp. 265-272, 1996.

[15] M. R. Coleman and W. J. Koros, "Isometric polyimides based on fluorinated dianhydrides and diamines for gas separation applications," Journal of Membrane Science, vol. 50, no. 3, pp. 285-297, 1990.

[16] M. K. Ghosh and K. L. Mittal, Polyimides: Fundamentals and Applications, vol. 36, CRC Press, New York, NY, USA, 1996.

[17] T. H. Kim, W. J. Koros, and G. R. Husk, “Temperature effects on gas permselection properties in hexafluoro aromatic polyimides," Journal of Membrane Science, vol. 46, no. 1, pp. 43-56, 1989.

[18] B. Xing and J. J. Pignatello, "Dual-mode sorption of low-polarity compounds in glassy poly (vinyl chloride) and soil organic matter," Environmental Science \& Technology, vol. 31, no. 3, pp. 792-799, 1997.

[19] R. M. Barrer, "Diffusivities in glassy polymers for the dual mode sorption model," Journal of Membrane Science, vol. 18, pp. 2535, 1984.

[20] E. Glueckauf, "The influence of ionic hydration on activity coefficients in concentrated electrolyte solutions," Transactions of the Faraday Society, vol. 51, pp. 1235-1244, 1955.

[21] T. Hirose, Y. Mi, S. A. Stern, and A. K. S. Clair, "The solubility of carbon dioxide and methane in polyimides at elevated pressures," Journal of Polymer Science B: Polymer Physics, vol. 29, no. 3, pp. 341-347, 1991. 

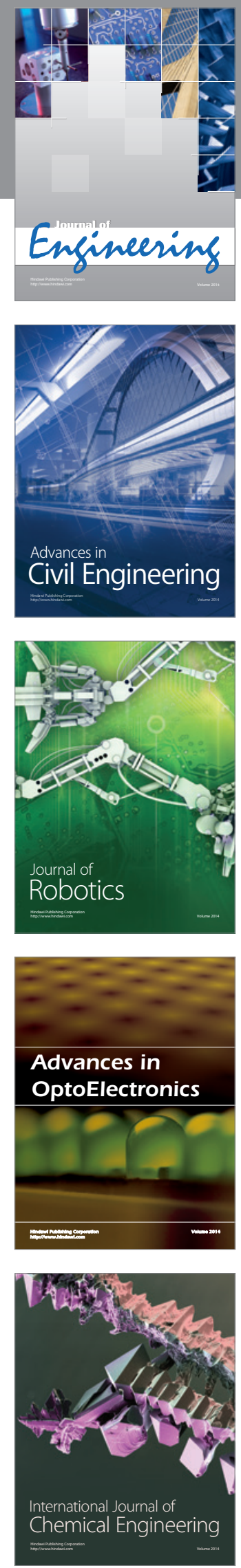

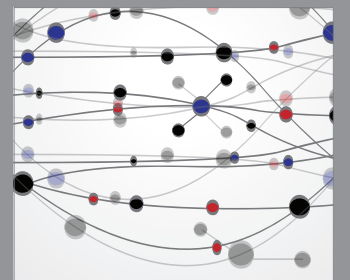

The Scientific World Journal
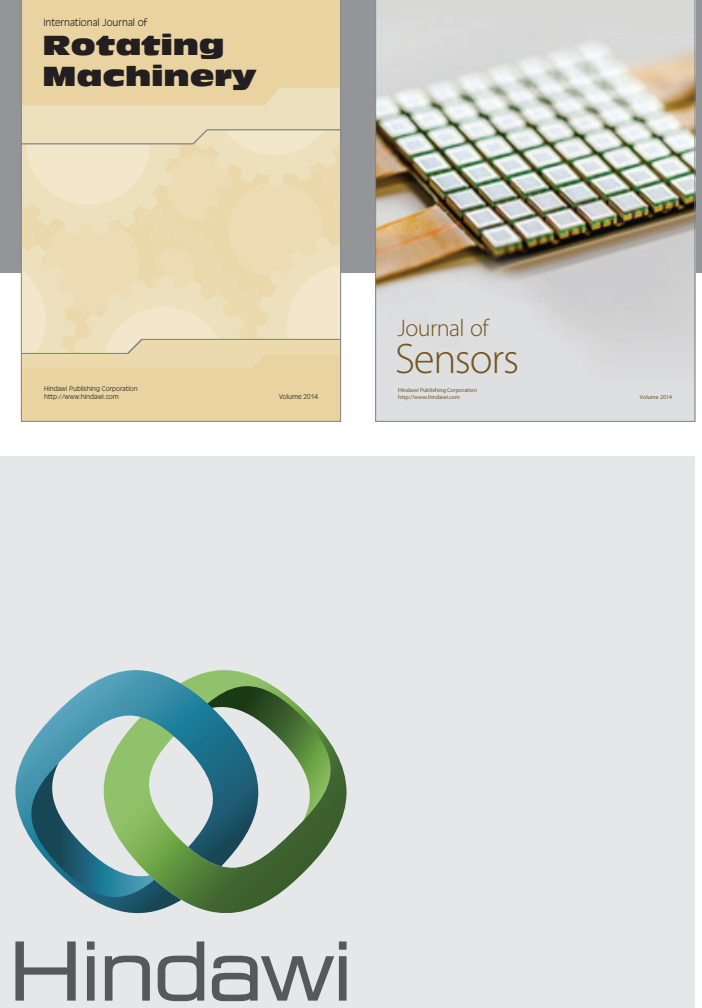

Submit your manuscripts at http://www.hindawi.com
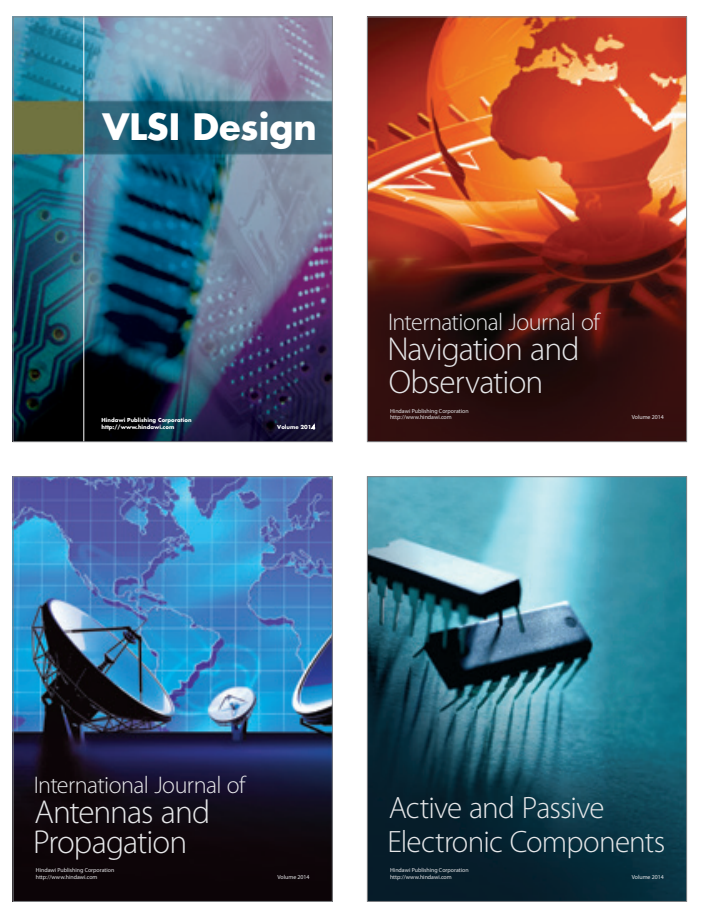
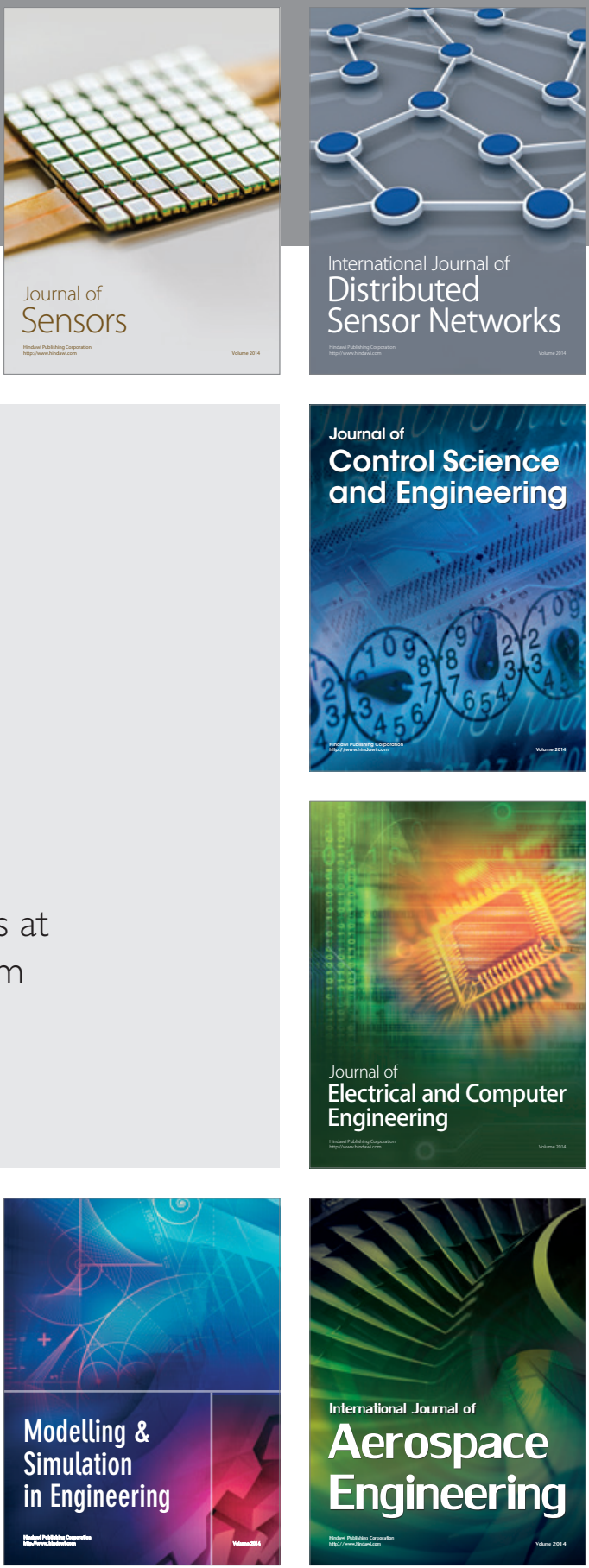

Journal of

Control Science

and Engineering
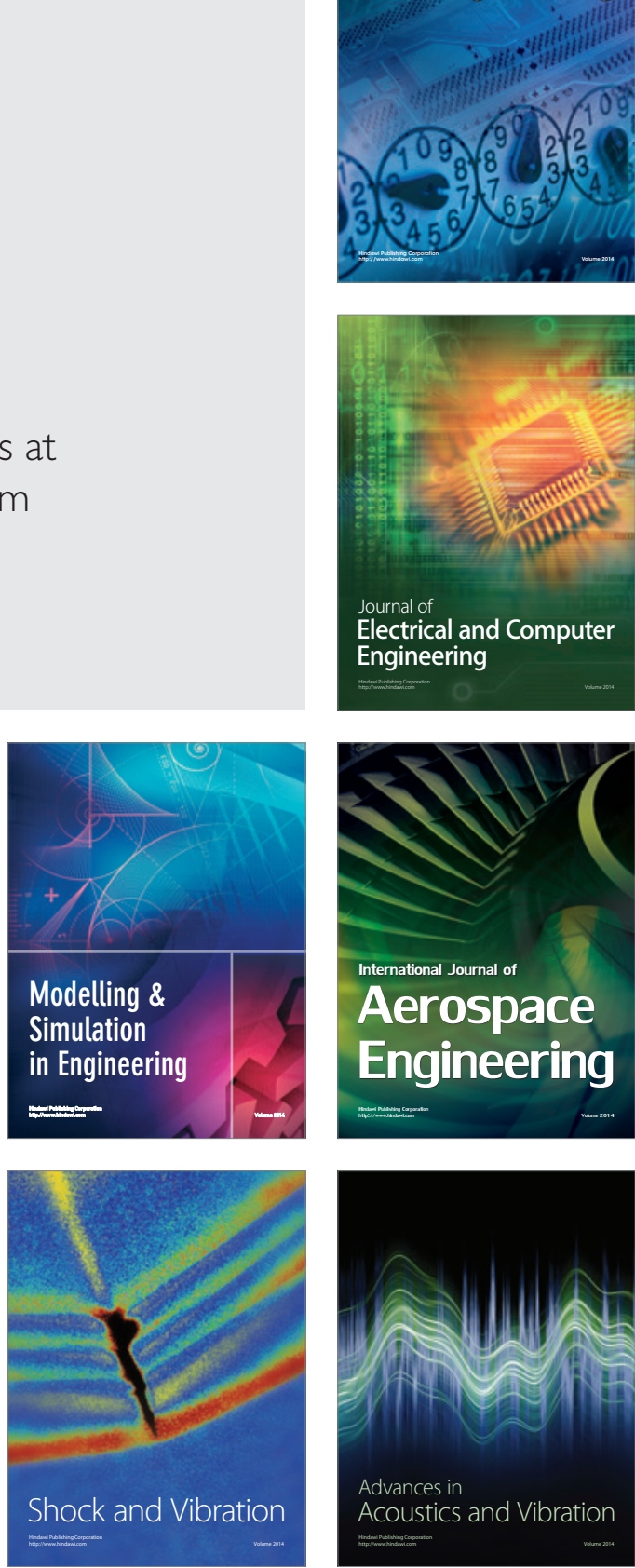